\title{
A study in intracardiac conduction 'Normalization' of bundle-branch block by fusion with ventricular escape beats
}

\author{
J. B. Witcombe ${ }^{1}$ and L. Schamroth \\ From the Departments of Medicine, the General Hospital, Baragwanath Hospital, \\ and the University of the Witwatersrand, fohannesburg, S.A.
}

\begin{abstract}
A case of high-grade AV block is described. Conducted sinus impulses are associated with complete or incomplete left bundle-branch block. The rhythm is complicated by ventricular escape beats arising in the left ventricle distal to the block. Synchronous invasion of the ventricles by the sinus and ventricular escape impulses results in a ventricular fusion complex which 'normalizes' the bundle-branch block pattern.
\end{abstract}

A ventricular fusion beat reflects the simultaneous activation of the ventricles by two impulses - usually a sinus impulse and an ectopic ventricular impulse - each activating part of the ventricles. The resulting QRS complex has a configuration that is in between that of the 'pure' uncomplicated sinus beat, and the 'pure' uncomplicated ectopic ventricular beat. If, however, the sinus beat is conducted with a bundle-branch block pattern, and it forms a fusion beat with a ventricular ectopic impulse originating in the same ventricle as the bundle-branch block, the bundle-branch block pattern is 'normalized'. This is because the ectopic ventricular impulse activates the region beyond the block, and the simultaneous activation of the ventricles by both impulses results in normal or near-normal activation (see Fig. 3A). This normalization of the bundlebranch block pattern has been produced experimentally by fusion with end-diastolic ventricular extrasystoles (Bisteni et al., 1960). The phenomenon has also been observed clinically when left ventricular end-diastolic extrasystoles occurred in bigeminal rhythm during sinus rhythm with left bundle-branch block (Schamroth and Chesler, I962). Here, the resulting normalization of alternate QRS complexes simulated $2:$ I left bundle-branch block. 1 Present address: Department of Radiology, The
Radcliffe Infirmary, Oxford.
The following case illustrates high-grade AV block with the conducted sinus beats showing either an incomplete or complete left bundle-branch block pattern. The intermittent AV block leads to ventricular escape, and the idioventricular escape focus is located in the left ventricle. Occasional ventricular fusion between the idioventricular escape impulse and the conducted sinus impulse results in normalization of the bundle-branch block pattern.

\section{Case report}

An obese 59-year-old woman presented with repeated syncopal attacks. Physical examination, apart from the finding of a slow irregular pulse, was essentially normal.

Fig. I (a continuous strip of lead VI) shows high-grade AV block with ventricular escape. This is reflected in the second half of the lower strip which shows complete AV dissociation between the sinus $P$ waves and the bizarre QRS complexes produced by the slow discharge of an idioventricular pacemaker. The PP intervals range from $0.73 \mathrm{sec}$ to $0.76 \mathrm{sec}$, representing a sinus rate of 79 to 82 beats a minute. The RR interval of the idioventricular escape rhythm is $I .33 \mathrm{sec}$, representing an idioventricular rate of 45 beats a minute. The QRS complexes of the idioventricular rhythm have a right bundle-branch block pattern, indicating that their origin is in the left ventricle. The Ist, $3 \mathrm{rd}$, and 5th $\mathrm{QRS}$ complexes in the upper strip represent ventricular escape beats arising from the same focus. The 2nd, $4^{\text {th }}$, 6th, and $7_{\text {th }}$ QRS complexes of the tracing 
represent conducted sinus beats. The $P R$ intervals of these beats measure $0.16 \mathrm{sec}$. The 2nd and 4 th QRS complexes reflect incomplete left bundlebranch block; the 6th QRS complex reflects complete left bundle-branch block; the 7th QRS complex has a normal configuration, but represents a ventricular fusion complex produced by partial activation of the ventricles by the conducted sinus impulse, and partial activation of the ventricles by the ectopic ventricular pacemaker.

Fig. 2 (strips of standard lead I, aVR, and aVF) shows the same manifestations. Sinus impulses conducted with complete left bundle-branch block are present in standard lead I (Ist QRS complex) and aVR (2nd QRS complex). Incomplete left bundle-branch block is represented by the $4^{\text {th }} Q R S$ complex in lead $I$, the 5 th QRS complex in aVR, and the 2nd, 4th, and 6th QRS complexes in aVF. The and QRS complex in standard lead $I$, and the 3 rd QRS complex in aVR, represent fusion beats and reflect normalization of the left bundle-branch block pattern. The remaining QRS complexes in the three leads represent ventricular escape beats. The rhythm in lead aVF is an uncomplicated escape-capture bigeminy (Bradley and Marriott, 1958): the escape of an idioventricular pacemaker followed by the 'capture' of the ventricles by the sinus impulse.

It is noteworthy that the ventricular fusion beats follow only the complexes with complete left bundle-branch block. The uncomplicated escape beats follow only the complexes with incomplete left bundle-branch block. The escape beats occur after an interval of $1 \cdot 36 \mathrm{sec}$ from the beginning of the QRS complexes with an incomplete left bundle-branch block pattern. In contrast to this, the escape beats which result in ventricular fusion occur after an interval of $1.46 \mathrm{sec}$ from the beginning of the QRS complexes with a complete left bundle-branch block pattern.

\section{Discussion}

Mechanism of normalization The sinus impulses are either blocked within the left bundle-branch, or conducted through it with delay. The idioventricular escape impulses arise in the left ventricle distal to the block. If, under these circumstances, the impulses from both pacemakers fortuitously invade the ventricles synchronously or at nearly the same time, the left ventricle distal to the block is activated by the idioventricular escape impulse whereas the rest of the ventricular myocardium is activated by the sinus impulse (Fig. 3A). This combined depolarization closely resembles depolarization of an uncomplicated normally conducted sinus impulse. This form of fusion complex thus manifests normalization of the bundlebranch block pattern.

'Linkage' of normalizing ventricular fusion to preceding complete left bundle-

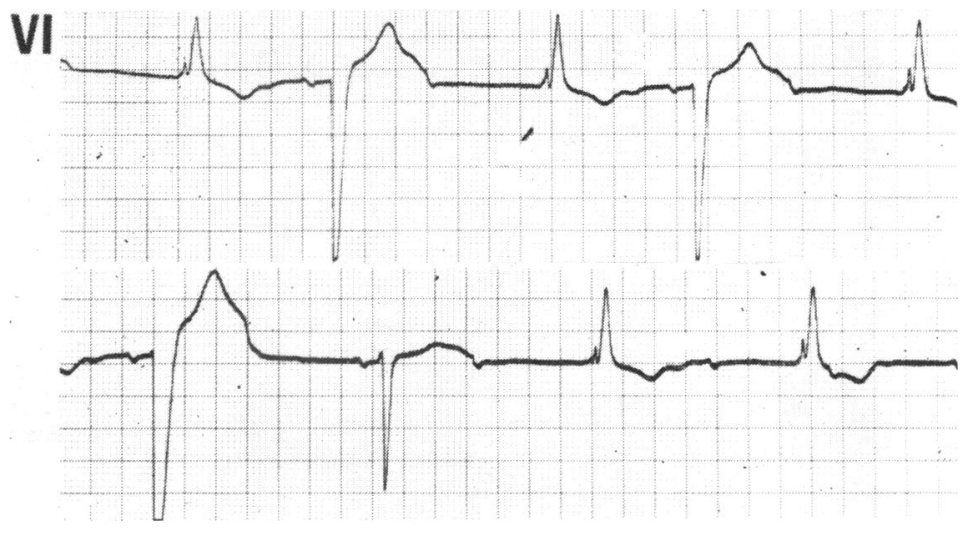

FIG. I Electrocardiogram (continuous strip

of lead $V_{I}$ ) showing high-grade $A V$ block, complete and incomplete left bundle-branch

block, ventricular escape beats of $R B B B$

pattern, and a normalizing fusion beat.

branch block The normalizing ventricular fusion complex occurs only after a sinus beat conducted with complete left bundle-branch block. The reason is as follows.

In the presence of a basic 2: I AV block, a QRS complex of a conducted sinus impulse will be recorded after an interval equivalent to 2 PP intervals from the preceding QRS complex (assuming constant conduction times). This is illustrated in Fig. 4A where the PP interval measures $0.73 \mathrm{sec}$, and the second QRS complex is inscribed at an interval of I.46 sec $(2 \times 0.73 \mathrm{sec})$ after the first QRS complex. The PP intervals of the whole tracing ranged from 0.73 to $0.76 \mathrm{sec}$. Thus, the sinus QRS complex may be recorded between I.46 sec $(2 \times 0.73 \mathrm{sec})$ and $1.52 \mathrm{sec}$ $(2 \times 0.76 \mathrm{sec})$ after the preceding sinus beat.

FIG. 2 Electrocardiogram (strips of standard

lead $I, a V R$, and $a V F$ ) showing high-grade

$A V$ block, complete and incomplete left

bundle-branch block, ventricular escape beats, and normalizing ventricular fusion beats.

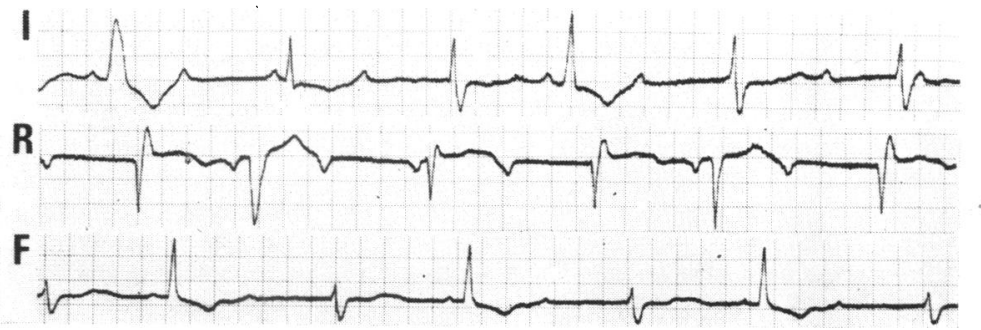




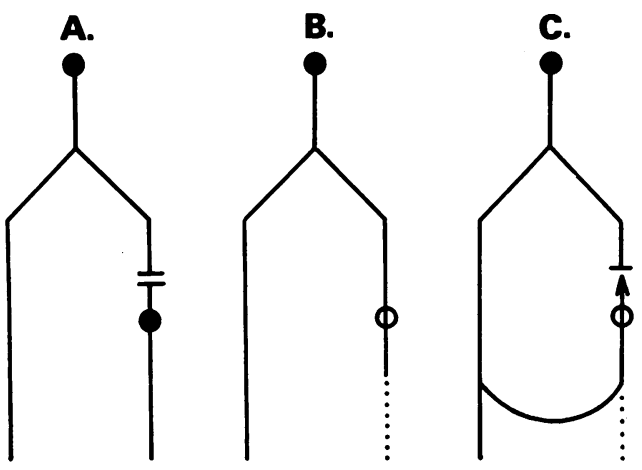

FIG. 3 Diagrams illustrating: $(A)$ the mechanism of normalization of left bundlebranch block by an idioventricular escape impulse arising in the left ventricle; $(B)$ discharge of the idioventricular pacemaker by a sinus impulse conducted with incomplete left bundle-branch block; $(C)$ discharge of the idioventricular pacemaker by a sinus impulse conducted with complete left bundle-branch block.

It is clear that, under these circumstances, ventricular fusion can only occur if the ventricular escape beat is initiated no earlier than I.46 sec after the preceding sinus beat, for only then will the sinus impulse and the idioventricular escape impulse invade the ventricles simultaneously. This is illustrated in Fig. 4C. If the ventricular escape impulse occurs sooner than $I .46 \mathrm{sec}$ after the preceding sinus beat, as is seen in Fig. $4 \mathrm{~B}$, where it occurs after only $I \cdot 36 \mathrm{sec}$, it is apparent that ventricular fusion cannot occur. For here, the ventricular escape impulse anticipates the sinus impulse and depolarizes the whole of the ventricular myocardium. The two impulses meet and interfere with each other within the atrioventricular node and lead to momentary AV dissociation. The ventricular escape beats follow incomplete left bundlebranch block at an interval of I.36 sec, and at an interval of $\mathrm{I} \cdot 46 \mathrm{sec}$ after complete left bundle-branch block. From the reasoning above, it is clear that ventricular fusion beats can only occur after the sinus beats conducted with complete bundle-branch block, for only then is the 'escape interval' $I \cdot 46 \mathrm{sec}$. The reason why the 'escape interval' varies and is longer when occurring after complete bundlebranch block is considered below.

Mechanism of varying escape intervals The cycle of an idioventricular escape pacemaker begins anew from the moment of its

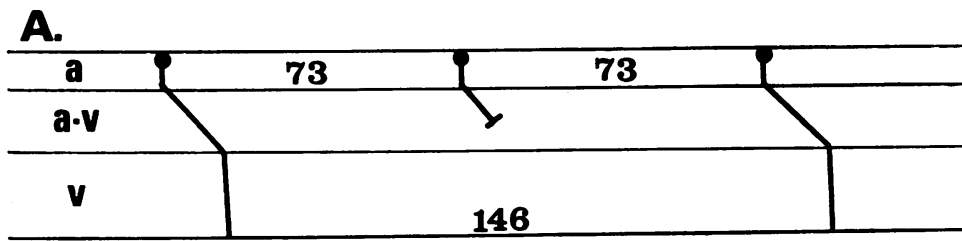

B.

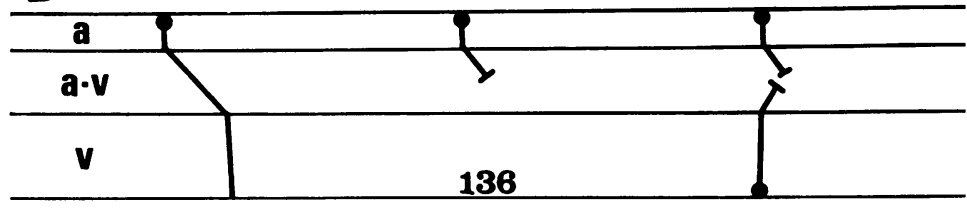

C.

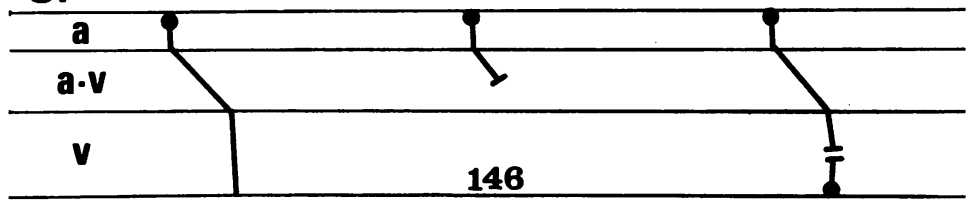

FIG. 4 Diagrams illustrating sinus rhythm conducted with 2:I $A V$ block $(A)$, complicated by ventricular escape $(B)$, and ventricular fusion $(C)$.

spontaneous or passive discharge. It will, for example, begin anew from the moment of its passive discharge by a sinus impulse. The idioventricular pacemaker in this case is situated in the left ventricle distal to the block. When the sinus impulse is conducted with incomplete left bundle-branch block, the idioventricular pacemaker is discharged by the sinus impulse which is conducted, albeit with delay, through the left bundle-branch (Fig. 3B). When the left bundle-branch block is complete, the idioventricular pacemaker can only be discharged by the sinus impulse which is conducted down the right bundlebranch and which can only reach the idioventricular pacemaker by a devious route - probably transseptal connexions (Fig. $3 \mathrm{C}$ ). When the sinus impulse depolarizes the left ventricle by this route, it takes slightly longer - about $0 \cdot 10$ sec ( $I \cdot 46 \mathrm{sec}$ minus $\mathrm{I} \cdot 36$ sec) - to reach the idioventricular pacemaker: The subsequent idioventricular discharge then also occurs 0.10 sec late, i.e. instead of occurring $I \cdot 36 \mathrm{sec}$ after the preceding sinus beat, it occurs after $I \cdot 46 \mathrm{sec}$. This longer escape interval enables the idioventricular pacemaker to activate the ventricles simul- 
taneously with the next sinus impulse and a fusion complex ensues.

Site of idioventricular pacemaker The true idioventricular pacemaker cycle, as shown by the $R R$ interval of the uncomplicated escape rhythm (see lower strip of Fig. I) measures $I \cdot 33 \mathrm{sec}$. The escape interval after incomplete left bundle-branch block, that is, the interval from the $R$ wave of the sinus impulse conducted with incomplete left bundle-branch block to the $R$ wave of the following idioventricular escape impulse, measures I. $36 \mathrm{sec}$. Thus, the idioventricular pacemaker is discharged by the sinus impulse conducted with incomplete left bundlebranch block $0.03 \mathrm{sec}$ after the beginning of the sinus QRS complex ( $\mathrm{I} \cdot 36 \mathrm{sec}$ minus $\mathrm{I} \cdot 33$ $\sec =0.03 \mathrm{sec}$ ). The escape interval following complete left bundle-branch block measures I.46 sec and therefore the idioventricular pacemaker is discharged $0.13 \mathrm{sec}$ after the beginning of the sinus QRS complex ( $\mathrm{I} \cdot 46$ sec minus $\mathrm{I} \cdot 33 \mathrm{sec}=0.13 \mathrm{sec}$ ). The very late discharge of the idioventricular pacemaker following a sinus impulse conducted with complete left bundle-branch block, and the earlier discharge by the sinus impulse conducted with incomplete left bundle-branch block suggests that the idioventricular pacemaker is situated just distal to the blocked region of the left bundle-branch (as depicted in Fig. 3A).

We are indebted to the Photographic Department, Department of Medicine, University of the Witwatersrand, for the photographic reproductions.

\section{References}

Bisteni, A., Sodi-Pallares, D., Medrano, G. A., and Pileggi, F. (1960). A new approach for the recognition of ventricular premature beats. American fournal of Cardiology, 5, 358.

Bradley, S. M., and Marriott, H. J. L. (1958). Escapecapture bigeminy. Report of a case of $\mathrm{A}-\mathrm{V}$ dissociation initiated by $2:$ I S-A block with resulting bigeminal rhythm. American fournal of Cardiology, I, 640 .

Schamroth, L., and Chesler, E. (1962). Simulated 2: I left bundle-branch block. Normalization of the left bundle-branch block pattern by ventricular extrasystoles. Circulation, 25, 395.

Requests for reprints to Dr. J. B. Witcombe, Department of Radiology, The Radcliffe Infirmary, Oxford.

\section{Notice}

\section{International Society of Cardiology}

\section{Scientific Council on Epidemiology and Prevention}

The Council on Epidemiology and Prevention, International Society of Cardiology, announces its Fifth Ten-Day International Teaching Seminar on Cardiovascular Epidemiology, 24 September-6 October 1972, in Singapore. Up to 35 Fellows can be accepted. Final acceptance is by the Council's Seminar Committee. Nominees should be at the postdoctoral level, with some residency training or its equivalent, and be interested in cardiovascular epidemiology. Limited funds may be available to pay for room and board during the Seminar and to give partial assistance with travel costs for accepted Fellows. Fluency in English is an absolute essential. Nominations should be submitted by chiefs of departments or institutions, and should be accompanied by a personal letter of application for the nominee, together with his curriculum vitae. The deadline for applications is I April 1972. They should be sent to Jeremiah Stamler, M.D., Secretary, Council on Epidemiology and Prevention, ISC, Room LL 139, Chicago Civic Center, Chicago, Illinois, 60602, U.S.A. 